\title{
Sul comportamento delle serie di potenze sugli archi di regolarità.
}

\author{
Memoria di Delfina Roux a Milanol (1).
}

Sunto. - Per ogni serie di potenze e le relative somme pareiali

$$
f(x)=\sum_{k=0}^{\infty} a_{k} x^{k} \quad f_{n}(x)=\sum_{k=0}^{n} a_{k} x^{k}
$$

un teorema di Fatou-Riesz assicura che $\mathrm{f}_{\mathrm{n}}(\mathrm{x}) \rightarrow \mathrm{f}(\mathrm{x})$ per $\mathrm{n} \rightarrow+\infty$ uniformemente sopra ogni arco di regolarità della circonferenza di convergenza $|\mathrm{x}|=1$, se $\mathrm{a}_{\mathrm{n}} \rightarrow 0$. Nella presente nota si stabiliscono due teoremi che dànno condizioni sufficienti perchè $\mathrm{f}_{\mathrm{n}_{\mathrm{h}}}(\mathrm{x}) \rightarrow \mathrm{f}(\mathrm{x}),\left(0=\mathrm{n}_{0}<\mathrm{n}_{1}<\ldots<\mathrm{n}_{\mathrm{h}}<\ldots\right)$, per $\mathrm{h} \rightarrow+\infty$ uniformemente su ogni tale arco. Nella condizione si tiene anche conto dell andamento di $\left|\mathrm{n}_{\mathrm{h}+1}-\mathrm{n}_{\mathrm{h}}\right|$ e della collocazione dei punti singolari.

1. E ben noto il teorema di P. FAtou secondo il quale una serie di potenze

$$
f(x)=\sum_{k=0}^{\infty} a_{k} x^{k}
$$

avente raggio $d i$ convergenza 1 , converge in ogni punto di regolarita del cerchio di convergenza, se $a_{n} \rightarrow 0$ per $n \rightarrow+\infty\left({ }^{2}\right)$. Anzi, M. Rresz dimostrò che tale convergenza è uniforme su ogni "arco di regolarità " (ciò̀ su ogni arco i cui punti, compresi gli estremi, sono regolari per l'elemento analitico $f(x)$ di centro $O)\left({ }^{3}\right)$.

Anzichè considerare la successione completa $\left\{f_{n}(x)\right\}: f_{0}(x), f_{1}(x), \ldots, f_{n}(x), \ldots$, con $f_{n}(x)=\sum_{k=0}^{n} a_{k} x^{k}$, fissiamo l'attenzione su una successione parziale

$$
\left\{f_{n_{h}}(x)\right\}: f_{n_{0}}(x), f_{n_{i}}(x), \ldots, f_{n_{h}}(x), \ldots, \quad\left(0=n_{0}<n_{i}<\ldots<n_{h}<\ldots\right)
$$

e poniamoci i seguenti quesiti :

10) Quale è una condizione sufficiente atta a garantire la convergenza uniforme di questa successione $\left\{f_{n_{k}}(x)\right\}$ su ogni arco di regolarità?

(1) Lavoro eseguito nell' Istituto Matematico della Università di Milano.

$\left(^{2}\right)$ P. Fatou, Séries trigonométriques et séries de Taylor, "Acta matematica", 30, p. 389, (1906).

( $\left.{ }^{3}\right)$ M. Riesz, Uber einen Sate des Herrn Fratou, "Journal für die reine und angewandte Mathematikn, 140, pp. 89-49, (1911).

M. Riesz, Nener Beweis des Eratouschen Satzes, Nachrichten von der Königlichen Gesellschaft der Wissenschaften zu Göttingen, mathematisch-physicalische Klasse, Jahrgang 1916, pp. 62-65.

Vedi anche per es. E. Landau, Ergebnisse der Funktionentheorie, 2. Aufl., Berlin, 1929, pp. $73-76$. 
20) Quale è l'influenza della densità della successione parziale $\left\{n_{h}\right\}$ cioè dell' andamento della successione $\left\{n_{h+1}-n_{h}\right\}$, sulla condizione stessa?

$\left.3^{\circ}\right)$ Quale è l'influenza della distanza fra le singolarità della funzione $f(x)$ e l'arco di regolarità, sullo scarto della convergenza medesima?

2. È evidente che, se su un arco di regolarità vale, uniformemente rispetto a $x$,

$$
f_{n}(x) \rightarrow f(x) \text { per } h \rightarrow+\infty
$$

allora, sullo stesso areo, risulta (ancora uniformemente rispetto a $x$ ) :

$$
f_{n_{h}}(x)-f_{n_{u}}(x) \rightarrow 0 \text { per } u \leqq h, u \rightarrow+\infty, h \rightarrow+\infty .
$$

Il caso elassico di FATOU-RIESz sussiste in conseguenza dal fatto che, posto $|x|=r$ e

risulta :

$$
\varepsilon_{u}=\max \left(\left|a_{u+1}\right|,\left|a_{u+2}\right|, \ldots\right)
$$

$$
\left|\frac{f_{m}(x)-f_{n}(x)}{x^{n}}\right| \leqq \varepsilon_{n} r\left(1+r+r^{2}+\cdots+r^{m-n-1}\right)=\varepsilon_{\prime \prime} r \frac{1-r^{m-n}}{1-r}
$$

con $\varepsilon_{n} \rightarrow 0$ per $n \rightarrow+\infty$.

Nel nostro caso la condizione è analoga e sussiste il seguente

TEOREMA I. - Poniamo $|\mathrm{x}|=\mathrm{r}$ e sia verificata la seguente condiaione:

(A) Per $\mathrm{u} \leqq \mathbf{h}, \mathbf{u} \rightarrow+\infty, \mathrm{h} \rightarrow+\infty$, si abbia, uniformemente rispetto a. $\mathrm{x}$, in un campo $\mathrm{C}$ :

$$
\begin{aligned}
& (1-r) \frac{f_{n_{h}}(x)-f_{n_{u}}(x)}{x^{n_{u}}} \rightarrow 0, \text { quando } \quad r<1 \\
& (r-1) \frac{f_{n_{h}}(x)-f_{n_{u}}(x)}{x^{n_{h}}} \rightarrow 0, \text { quando } \quad r>1 .
\end{aligned}
$$

Allora :

$$
f_{n_{h}}(x) \rightarrow f(x) \text { per } h \rightarrow+\infty
$$

uniformemente su ogni arco di regolarità interno $a \mathrm{C}$.

Sia $A_{\delta}$ l'insieme (chiuso) dei punti appartenenti al contorno della stella di regolarità (secondo G. MITTAG LEFFLer) di centro $x=0$ e aventi modulo $\leqq 1+2 \delta$, e $\bar{A}_{\delta}$ l'insieme costituito dai punti $x$ che distano da $A_{\delta}$ per meno di $\delta$. Sussiste il seguente:

Teorema II. - Supponiamo che esistano tre costanti positive $\delta_{0}, \mathrm{~K}, \omega$, tali che, per $\delta \leqq \delta_{0}$, si abbia:

$$
|f(x)|<\frac{K}{\delta \omega}
$$

per ogni punto $\mathrm{x}$ interno a $\mathrm{C}$ ed esterno ad $\overline{\mathrm{A}}_{\delta}$, e tale che $|\mathrm{x}| \leqq 1+\delta$. Allora, se ̀̀ soddisfatta la condizione (A), esiste una successione $\left\{\eta_{h}\right\}$, con $\eta_{h} \rightarrow 0$ per $\mathrm{h} \rightarrow+\infty$, tale che risulti:

$$
\left|f(x)-f_{n_{h}}(x)\right|<\frac{\eta_{h}}{(\gamma+\delta) \delta \omega+2}
$$


sopra ogni arco di regolarità di ampiezza $\gamma$, interno a $\mathrm{C}$ ed esterno ad $\overline{\mathrm{A}_{\delta}}$, la cui distanza dal contorno di $\mathrm{C}$ è per lo meno $\delta$.

La successione $\left\{\eta_{h}\right\}$ è indipendente da $\mathrm{x}$ e dall' arco di regolarità.

3. 亡̀ opportuno sostituire alla condizione sufficiente (A) altre condizioni che riguardino il comportamento dello scarto $f_{n_{h+1}}(x)-f_{n_{h}}(x)$ di due somme parziali contigue della successione $\left\{f_{n_{u}}(x)\right\}$ considerata.

Poniamo $g_{u}=\operatorname{Min}\left(n_{u}-n_{u-1}, n_{u+1}-n_{u}, \ldots\right)$. La condizione (A) è verificata, e quindi vale il Teorema I, quando ò verificata la seguente condizione:

$$
\frac{f_{n_{h+1}}(x)-f_{n_{h}}(x)}{x_{h}^{n_{h}}}-0, \text { per } h \rightarrow+\infty \text {, quando } r<1
$$

$$
\frac{f_{n_{h+1}}(x)-f_{n_{h}}(x)}{g_{h+1} x_{h}^{n_{h}}}-0, \text { per } h \rightarrow+\infty, \text { quando } \mathrm{r}>1 \text {. }
$$

uniformemente rispetto a $\mathrm{x}$ nel campo $\mathrm{C}$.

Alla condizione (B) si può dare un'altra forma; a tal uopo introduciamo le seguenti notazioni :

$$
\begin{aligned}
f_{n_{h+1}}-f_{n_{k}}(x) & =a_{n_{h}+1} x^{n_{h}+1}+a_{n_{h}+2} x^{n_{h}+2}+\ldots+a_{n_{h+1}} x^{n_{h+1}} \\
& =x^{n_{h}}\left\{a_{n_{h}+1} x+a_{n_{h}+2} x^{2}+\ldots+a_{n_{h+1}} x^{n_{h+1}}{ }^{n_{h}}\right\} \\
& =x^{n_{h} P_{h+1}(x)}
\end{aligned}
$$

dove $P_{k+1}(x)$ è un polinomio di grado $d_{k+1}=n_{k+1}-n_{h}$. La condizione (B) assume allora la forma:

$$
\begin{aligned}
& P_{h}(x) \rightarrow 0, \text { per } h \rightarrow+\infty, \text { quando } r<1 \\
& \frac{P_{h}(x)}{g_{h}}-0, \text { per } h \rightarrow+\infty, \text { quando } r>1,
\end{aligned}
$$

uniformemente rispetto a $\mathrm{x}$ nel campo $\mathrm{C}$.

Quando $\left\{g_{h}\right\}$ (che risulta non decrescente) è limitata, la condizione (B) si riduce a:

$\mathrm{P}_{\mathrm{h}}(\mathrm{x})-0$ per $\mathrm{h} \rightarrow+\infty$, uniformemente rispetto a $\mathrm{x}$ nel campo $\mathrm{C}$.

\section{Osservazioni.}

I. - Nel caso in cui la successione $\left\{n_{h}\right\}$ sia quella degli interi naturali, il Teurema I coincide con quello classico di FAtod-RIEsz. Infatti, in questo caso $n_{h}=h, n_{h+1}-n_{h}=1, g_{h}=1, P_{h}(x)=a_{h} x, a_{h} x \rightarrow 0$ uniformemente per $|x| \leq 1+k$ (qualunque sia $k$ ).

II. - Il Teorema I è una ovvia conseguenza del Teorema II, quando sia fissato un arco di regolarità (quindi fissati $\gamma$ e $\delta$ ).

III. - Esistono successioni di polinomi in cui il coefficiente del termine generale non tende a 0 e per $\mathrm{i}$ quali sono verilicate le ipotesi del Teorema. Si considerino, ad esempio, i polinomi :

$$
P_{h}(x)=(1+x)^{2 h} /\left(\begin{array}{c}
2 h \\
h
\end{array}\right) ;
$$


i loro soefficienti hanno il valore assolnto $\leqq 1$ e, per ogni $h$, esiste un coefficiente $=1$; inoltre, essendo

$$
1 /\left(\begin{array}{c}
2 h \\
h
\end{array}\right)=\frac{(h !)^{2}}{(2 h) !} \sim \frac{\sqrt{2 \pi h}}{e^{22^{2 h}}}
$$

si ha che $P_{h}(x) \rightarrow 0$ uniformemente rispetto a $x$ in ogni cerchio interno al cerchio di centro -1 e raggio 2 . Cioè i polinomi $P_{h}(x)$ soddisfano la condizione $\left(B^{\prime}\right)$ nel cerchio di centro -1 e raggio $2-\eta$ (per ogni $\eta>0$ ).

Osserviamo anche che la condizione (B') è meno restrittiva della condizione $P_{h}(x) \rightarrow 0$ uniformemente per $h \rightarrow \infty$. Per esempio, i polinomi

$$
\bar{P}_{h}(x)=\frac{x^{h}}{h}+(1+x)^{2^{h+1}} /\left(\begin{array}{c}
2^{h+1} \\
2^{h}
\end{array}\right)
$$

soddisfano alla condizione $\left(B^{\prime}\right)$ nel cerohio di centro -1 e raggio $2-\eta$ (per ogni $\eta$ positivo), mentre soddisfano la condizione $\bar{P}_{h}(x) \rightarrow 0$ uniformemente rispetto a $x$ per $h \rightarrow+\infty$, nel campo $C$ costituito dai punti comuni ai due cerchi $|x| \leqq 1 ;|x+1| \leqq 2-\eta$.

IV. - Se l'elemento analitico $f(x)$ possiede sulla eirconferenza di convergenza solo singolarità non essenziali isolate fciò̀ solo poli è punti di diramazione algebroidi), è certamente possibile determinare le costanti $\delta_{0}, K, \omega$, in modo che, per $r \leqq 1+\delta_{0}$, valga la condizione (1) del Teorema II.

5. Da (B) segue (A).

Veniamo a dimostrare che $(B) \Longrightarrow(A)$. Consideriamo (B) nella forma (B'). Sia $\sigma_{u}$ l'estremo superiore dei valori

$$
\begin{aligned}
& \left|P_{u+1}(x)\right|, \quad\left|P_{u+2}(x)\right|, \ldots, \text { per } r<1, x \text { in } C \\
& \frac{\left|P_{u+1}(x)\right|}{g_{u+1}}, \frac{\left|P_{u+2}(x)\right|}{g_{u+2}}, \ldots, \text { per } r>1, x \text { in } C .
\end{aligned}
$$

Per le ipotesi fatte, $\sigma_{u}$ esiste finito e $\sigma_{u} \rightarrow 0$ per $u \rightarrow+\infty$.

Sia $r<1$. Si ha:

$$
\begin{aligned}
& \left|f_{n_{k}}(x)-f_{u_{u}}(x)\right|=\left|a_{n_{u}+1} x^{n_{u}+1}+a_{n_{u}+2} x^{n}{ }_{u}+2+\ldots+a_{n_{k}} x^{n_{h}}\right| \\
& \leqq \boldsymbol{r}^{n_{u}}\left|P_{u+1}(x)\right|+\boldsymbol{r}^{n_{u+1}}\left|P_{u+2}(x)\right|+\ldots+\boldsymbol{r}^{n_{h-1}}\left|P_{h}(x)\right| \\
& \leqq \sigma_{u} r^{n_{u}}\left\{1+r^{n_{u+1}-n_{u}}+r^{n_{u+2}}-n_{u}+\ldots+r^{n_{h-1}-n_{u}}\right\} \\
& <\sigma_{u} r^{n_{u}}\left\{1+r+r^{2}+\ldots\right\} \\
& <\sigma_{u} \frac{r^{n_{u}}}{1-r} \text {. }
\end{aligned}
$$

Resta così dimostrato che $(\mathrm{B}) \Rightarrow(\mathrm{A}) \mathrm{p}(\mathrm{r} r<1$. 
Sia $r>1$. Si ha :

$$
\begin{aligned}
\left|f_{n_{k}}(x)-f_{n_{u}}(x)\right| & =a_{n_{u}+1} x^{n_{u}+1}+a_{n_{u}+2^{2}} x^{n_{u}+2}+\ldots+a_{n_{h}} x^{n_{h}} \mid \\
& \leqq r_{u}\left|P_{u+1}(x)\right|+r_{u+1}^{n_{u}}\left|P_{u+2}(x)\right|+\ldots+r^{n_{h-1}}\left|P_{h}(x)\right| \\
& \leqq r^{n_{u}} \frac{\left(1+r+\ldots+r^{a_{u+1}-1}\right)}{g_{u+1}}\left|P_{u+1}(x)\right|+\ldots+r^{n_{h-1}} \frac{\left(1+r+\ldots+r^{a_{h}-1}\right)}{g_{h}}\left|P_{h}(x)\right| \\
& \leqq \sigma_{u}\left\{r^{n_{u}}+r^{n_{u}+1}+r_{u}^{n_{u}+2}+\ldots r^{n_{h}-1}\right\} \\
& \leqq \sigma_{u}\left\{1+r+r^{2}+\ldots+r^{n_{h}-1}\right\} \\
& \leqq \sigma_{u} \frac{r^{n_{h}}-1}{r-1} \\
& <\sigma_{u} \frac{r^{n_{h}}}{r-1}
\end{aligned}
$$

ciò̀ la condizione (A) per $r>1$.

\section{Dimostrazione dei Teoremi I e II.}

Veniamo a dimostrare il Teorema II; il Teorema $I$ e, come abbiamo gia osservato, una conseguenza di questo.

Sia $r<1$. Diciamo $\varepsilon_{u}$ l'estremo superiore dei moduli

$$
\left(\begin{array}{ll}
1 & r
\end{array}\right)\left|\frac{f_{n_{n}}(x)-f_{n_{u}}(x)}{x^{n_{u}}}\right| ; h=u+1, u+2, \ldots ; x \text { in } C .
$$

Per l'ipotesi fatta $\varepsilon_{u}$ esiste finito e $\varepsilon_{u} \rightarrow 0$ per $u \rightarrow+\infty$. Inoltre risulta:

$$
(1-r)\left|\frac{f(x)-f_{n_{u}}(x)}{x^{n_{w}}}\right| \leqq \varepsilon_{u}
$$

e, scrivendo $h$ in luogo di $u$, abbiamo:

(3)

$$
\left|\frac{f(x)-f_{n_{h}}(x)}{x^{n_{h}}}\right| \leqq \frac{\varepsilon_{h}}{1-r}, r<1, x \text { in } C .
$$

Sia $r>1$. Allora :

$$
\begin{aligned}
\left|\frac{f(x)-f_{n_{h}}(x)}{x^{n_{h}}}\right| & =\left|\frac{f(x)-f_{n_{u}}(x)-f_{n_{h}}(x)+f_{n_{u}}(x)}{x^{n_{h}}}\right| \\
& \leqq \frac{|f(x)|+\left|f_{n_{u}}(x)\right|}{r^{n_{h}}}+\frac{\left|f_{n_{h}}(x)-f_{n_{u}}(x)\right|}{r^{n_{h}}} .
\end{aligned}
$$

Sia $\varepsilon_{u}^{\prime}$ il numero che, per $r>1$, ha significato analogo a quello di $\varepsilon_{u}$ per $r<1$; allora $\varepsilon_{u}^{\prime} \rightarrow 0$ per $u \rightarrow+\infty$ e

$$
\left|\frac{f(x)-f_{n_{h}}(x)}{x^{n_{h}}}\right| \leqq \frac{|f(x)|+\left|f_{n_{u}}(x)\right|}{r^{n_{h}}}+\frac{\varepsilon_{u}^{\prime}}{r-1} .
$$


Sia ora

$$
1<r \leqq 1+\frac{\delta_{0}}{2}=r_{0} ; \quad M_{u}(r)=\sum_{k=0}^{n_{u}}\left|a_{k}\right| r^{k} ; \quad S_{u}=\sum_{k=0}^{n_{u}}\left|a_{k}\right| .
$$

Risulta dalla (4), ricordando la (1):

$$
\left|\frac{f(x)-f_{n_{h}}(x)}{x^{n_{h}}}\right| \leqq \frac{K}{\delta r^{n_{h}}}+\frac{\mathbf{M}_{u t}(r)}{r_{h}^{n_{h}}}+\frac{\varepsilon^{\prime}}{r-1}
$$

Ma

$$
\begin{gathered}
r^{n_{h}}=\{1+(r-1)\}^{n_{h}} \geqq n_{h}(r-1) \\
\frac{M_{u}(r)}{r^{n_{h}}}=\frac{M_{u}(r)}{r^{n_{h}-n_{u} \cdot r^{n_{u}}} \leqq \frac{S_{u}}{r^{n_{h}-n_{u}}} \leqq \frac{S_{u}}{\left(n_{h}-n_{u}\right)(r-1)}} .
\end{gathered}
$$

Fissato $h$, risulta fissato $n_{h}$ : si scelga per $u$ il massimo intero per cai sono verificate le disuguaglianze: $n_{u} \leqq n_{h} / 2 ; S_{u} \leqq \sqrt{n_{h}}$. Per $h$ abbastanza grande $u$ d definito $\theta$ inoltre per $h \rightarrow+\infty$ risulta evidentemente $n_{u} \rightarrow+\infty$, $u \rightarrow+\infty$. In queste condizioni, dalla (5) risulta:

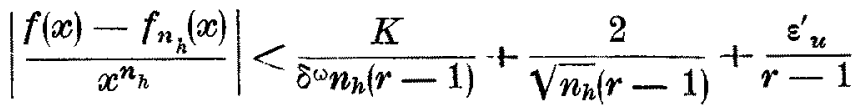

$$
\begin{aligned}
& \leqq \frac{1}{\delta \omega(r-1)}\left|\frac{K}{n_{h}}+\frac{2 \delta_{0} \omega}{\sqrt{n_{h}}}+\varepsilon^{\prime}{ }_{u} \delta_{0} \omega\right|
\end{aligned}
$$

ciò̀

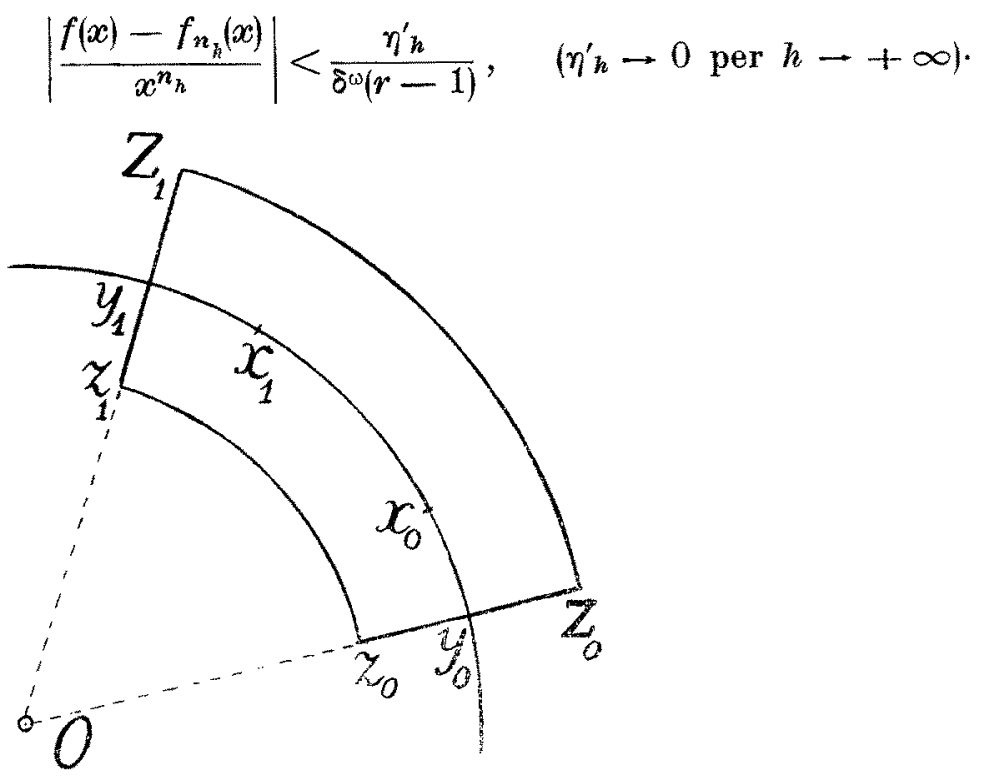

Sia $\widehat{x_{i}, x_{2}}$ un arco di regolarita di ampiezza $\gamma, \theta x_{0}$ il punto medio di $\widehat{x_{1}, x_{2}}$. Possiamo supporre $\delta \leqq \delta_{0} \leqq 2$. Ampliamo l'arco $\overparen{x_{0}, x_{1}}$ nell'arco $\overparen{y_{0}, y_{1}}$ 
aggiungendo un arco di ampiezza $\delta / 2$ da ambedue le parti. Denotiamo con $S_{\delta}$ il settore di corona circolare limitato dai raggi $z_{0} Z_{0}$ pel punto $y_{0}, z_{1} Z_{1}$ pel punto $y_{1}$, e dalle due circonferenze di centro 0 e raggio rispettivamente $1-\delta / 2$ e $1+\delta / 2$.

Consideriamo la funzione

$$
\varphi_{n_{h}}(x)=\frac{f(x)-f_{n_{h}}(x)}{x^{n_{h}}}\left(x-y_{0}\right)\left(x-y_{1}\right)
$$

essa è regolare in $S_{\delta}$ e, detto $\mu_{h}$ il suo massimo modulo sul contorno di $S_{\delta}$, pei punti $x$ dell' arco $x_{0}, x_{1}$ risulta:

e quindi, essendo $x \mid=1$

$$
\left|\varphi_{n_{h}}(x)\right| \leqq \mu_{n}
$$

$$
\left|f(x)-f_{n_{h}}(x)\right| \leqq \frac{\mu_{h}}{\left|x-y_{0}\right|\left|x-y_{1}\right|} .
$$

Al variare di $x$ sull'arco $\widehat{x_{0}, x_{1}}$, il denominatore assume il minimo modulo per $x=x_{0}$ e per $x=x_{1}$; e, poichè il rapporto fra un arco non superiore a $\pi$ e la sua corda non supera $\pi / 2$, tale minimo non è inferiore a:

$$
\left(\frac{\delta}{2} \cdot \frac{\gamma+\delta}{2}\right) \cdot \frac{2}{\pi}=\frac{\delta(\gamma+\delta)}{2 \pi}
$$

Ne segue, pei punti $x$ dell' arco $\overparen{x_{0}, x_{1}}$ :

$$
\left|f(x)-f_{n_{h}}(x)\right|<\frac{2 \pi \mu_{h}}{(\gamma+\delta) \delta}
$$

Si tratta di studiare $\mu_{h}$. Sull' arco $\overparen{z_{0}, z_{1}}$, (estremi inclusi), essendo $r=1-\frac{\delta}{2}$, per la $(3)$ risulta :

$$
\begin{aligned}
& \left|\frac{f(x)-f_{n_{h}}(x)}{x^{n_{h}}}\right|<\frac{2 \varepsilon_{h}}{\delta} \\
& \left|\varphi_{n_{h}}(x)\right|<\frac{2 \varepsilon_{h}}{\delta}\left|x-y_{0}\right|\left|x-y_{1}\right|<\frac{2 \varepsilon_{h}}{\delta} \cdot 2 \cdot 2
\end{aligned}
$$

pertanto:

$$
\left|\varphi_{n}(x)\right|<\frac{8 \varepsilon_{h}}{\delta} \text { sopra } \overparen{z_{0}, z_{1}} .
$$

Ancora per la (3), sui raggi $z_{9} y_{0}, z_{1} y_{1}$ (secondi estremi esclusi), abbiamo:

$$
\left|\varphi_{n_{h}}(x)\right|<\frac{\varepsilon_{h}}{1-r}\left|x-y_{0}\right|\left|x-y_{1}\right|<\frac{\varepsilon_{h}}{1-r}(1-r) \cdot 2
$$


e ciò̀

$$
\left|\varphi_{n_{h}}(x)\right|<2 \varepsilon_{h}, \quad \text { sopra } \overline{z_{1} y_{0}} \text { e } \overline{z_{1} y_{1}} \text {. }
$$

Per la (6), sui raggi $y_{0} Z_{0}, y_{1} Z_{1}$ (primi estremi esclusi), risulta:

$$
\left|\varphi_{n_{h}}(x)\right|<\frac{\eta_{h}^{\prime}}{\delta \omega(r-1)}\left|x-y_{0}\right|\left|x-y_{\imath}\right|<\frac{\eta_{h}^{\prime}}{\delta \omega(r-1)}(r-1) \cdot 2 r
$$

e quindi

$$
\left|\varphi_{n}(x)\right|<\frac{4 \eta^{\prime} h}{\delta \omega} \text { sopra } \overline{y_{0} Z_{0}} \text { e } \overline{y_{1} Z_{1}}
$$

Infine, sempre per la $(6)$, sull' arco $\overparen{Z_{0} Z_{1}}$, essendo $r=1+\delta / 2$, risulta:

$$
\left|\varphi_{n_{h}}(x)\right|<\frac{\eta_{h}^{\prime}}{\delta^{\omega} \cdot \frac{\delta}{2}}\left|x-y_{0}\right|\left|x-y_{1}\right|<\frac{2 \eta_{h}^{\prime}}{\delta \omega+1} \cdot 3 \cdot 3
$$

e quindi

$$
\left|\varphi_{n_{h}}(x)\right|<\frac{18 \eta^{\prime} h}{\delta \omega+1}, \text { sopra } \widehat{Z_{0} Z_{1}}
$$

Nei punti $x=y_{10}, x=y_{1}$, la $\varphi_{n_{h}}(x)$ risulta continua $e$ nulla.

Si ha allora, dalle (8), (9), (10), (11), che il massimo modulo $\mu_{h}$ della funzione $\varphi_{n_{h}}(x)$ sul contorno di $S_{\delta}$ non supera il massimo dei quattro numeri

$$
\frac{8 \varepsilon_{h}}{\delta} ; \quad 2 \varepsilon_{h} ; \quad \frac{4 \eta_{h}^{\prime}}{\delta \omega} ; \quad \frac{18 \eta_{h}^{\prime}}{\delta \omega+l} .
$$

Indichiamo allora con $\eta_{h} / 2 \pi$ il massimo numero fra $8 \varepsilon_{h} \delta_{0}{ }^{\omega} ; 2 \varepsilon_{h} \delta_{0}{ }^{\omega+1}$; $4 \eta_{h}^{\prime} \delta_{0} ; 18 \eta_{h}^{\prime}$ : è evidente che $\eta_{h}$ non dipende dall' arco $\widehat{x_{0}, x_{1}}$ e che $\eta_{h} \rightarrow 0$ per $h \rightarrow+\infty$.

Dalla (7) si ricava allora, pei punti $x$ dell'arco $\widehat{x_{0}, x_{1}}$, la disuguaglianza

$$
\left|f(x)-f_{n_{h}}(x)\right|<2 \pi \frac{\eta_{h}}{2 \pi \delta \omega+1} /(\gamma+\delta) \delta
$$

e quindi vale

$$
\left|f(x)-f_{n_{h}}(x)\right|<\frac{\eta_{h}}{(\gamma+\delta) \delta^{\omega+2}}
$$

per ogni $x$ sull'arco $\widehat{x_{0}, x_{1}}$, e $\eta_{h} \rightarrow 0$ per $h \rightarrow+\infty$.

Si può ripetere per l'arco $\widehat{x}_{0}, x_{2}$ il medesimo ragionamento, giungendo al medesimo $\eta_{h}$; la (12) vale uniformemente rispetto a $x$ sull'intero arco $x_{1}, x_{2}$.

Il Teorema II resta cosi dimostrato: 